\title{
Stage IC Uterine Sarcoma AJCC v7
}

National Cancer Institute

\section{Source}

National Cancer Institute. Stage IC Uterine Sarcoma A/CC V7. NCI Thesaurus. Code C87134.

Stage IC includes: T1C, N0, M0. T1 1: For adenosarcoma: tumor invades more than half of the myometrium. N0: No regional lymph node metastasis. M0: No distant metastasis. Stage IC does not apply for leiomyosarcoma and endometrial stromal sarcoma. (AJCC 7th ed.) 